Journal of Engineering and Applied Sciences 15 (1): 81-87, 2020

ISSN: 1816-949X

(C) Medwell Journals, 2020

\title{
Artificial Neural Networks in the Demand Forecasting of a Metal-Mechanical Industry
}

\author{
${ }^{1}$ Leandro L. Lorente-Leyva, ${ }^{1}$ Delio R. Patino-Alarcon, ${ }^{1}$ Yakcleem Montero-Santos, \\ ${ }^{1}$ Israel D. Herrera-Granda, ${ }^{2}$ Diego H. Peluffo-Ordonez, ${ }^{3}$ Arlys M. Lastre-Aleaga and \\ ${ }^{3}$ Alexis Cordoves-Garcia \\ ${ }^{1}$ Facultad de Ingenieria en Ciencias Aplicadas, Universidad Tecnica del Norte, \\ Ibarra, Ecuador \\ ${ }^{2}$ SDAS Research Group, Yachay Tech, Urcuqui, Ecuador \\ ${ }^{3}$ Facultad de Ciencias de la Ingenieria e Industrias, Universidad UTE, \\ Santo Domingo de los Tsachilas, Ecuador
}

\begin{abstract}
This research presents an application of artificial neural networks in demand forecasting by using MATLAB Software. Keeping in mind that in any planning process forecasts play a fundamental role, being one of the bases for; planning, organizing and controlling production. It gives priority to the most critical nodes and their key activities, so that, the decisions made about them will generate the greatest possible positive impact. The methodology applied demonstrates the quality of the solutions found which are compared with traditional statistical methods to demonstrate the value of the solution proposed. When the results show that the minimum quadratic error is reached with the application of artificial neural networks, a better performance is obtained. Therefore, a suitable horizon is established for the planification and decision making in the metal-mechanical industry for the use of artificial intelligence in the production processes.
\end{abstract}

Key words: Demand forecasting, artificial neural networks, metal-mechanical industry, production planning, industry, artificial intelligence

\section{INTRODUCTION}

In production planning problems, real situations are often uncertain. Due to faulty planning of the future state, it may not be fully known. This type of uncertainty (stochastic in nature) has traditionally been managed through probability theory and statistics.

Bellman and Zadeh (1970) call this type of variation stochastic uncertainty in contrast to the lack of precision present in the description of the semantic meaning of events, phenomena or sentences which they call fuzziness. Randomness or uncertainty is present in all areas in which human criteria, evaluation and decisions are important such as production planning.

An organization must plan production before it can carry it out. Without production planning it is likely that a company will not be able to produce enough to achieve an increase in future demand. Capacity may not be available at that time and sales may be lost (Lorente-Leyva et al., 2018).

Forecasting is the science and art of predicting future events. It may involve taking historical data and projecting it into the future with mathematical model, it may also be a subjective or intuitive prediction or it may involve a combination of these that is a mathematical model adjusted by the good judgment of an administrator.
Rarely is there a technique superior to the others what works best in a company under one set of conditions can be a complete disaster in another organization or even in a different department in the same way (Verma et al., 2017).

However, because effective strategic planning, both in the short and long term, depends on a forecast of demand. It is where the use of new techniques has raised satisfactory solutions such as the use of Artificial Neural Networks (ANN). Inspired by the biological model, they are generalizations of classic statistical models. Their novelty lies in sequential learning, the fact of using transformations of the original variables for prediction and non-linearity of the model. It allows learning in difficult contexts without specifying the formulation of a specific model.

In recent years, ANN's have become very popular in forecasting time series in a large number of areas including finance, power generation, medicine, water resources and environmental sciences, among other applications (Marroquin et al., 2009; Chen et al., 2012; Sarmiento and Soto, 2014 and Lorente-Leyva et al., 2018).

In order to make a forecast, it is common to require quantitative information on the behavior of demand through time that is a series of times, being the analysis of

Corresponding Author: Leandro L. Lorente-Leyva, Facultad de Ingenieria en Ciencias Aplicadas, Universidad Tecnica del Norte, Ibarra, Ecuador 
these statistical techniques the most used to estimate its behavior (Marroquin et al., 2009; Kim and Kim, 2016 and Verma et al., 2017). For many years, this type of analysis has been dominated using linear statistical methods that can be conveniently implemented; however, the existence of non-linear relationships between data can limit the application of these models. In practice it is very possible to find non-linear relationships in the data as in this case study. Therefore, it is necessary to use techniques capable of reflecting such behavior.

Several researchers have used different techniques to forecast demand (Kochak and Sharma, 2015) for the prediction of future data with afuzzy algorithm (Rubio et al., 2017). Forecasting tourism demand (Claveria and Torra, 2014). The urban water demand forecasting and uncertainty assessment with neural network models (Tiwari and Adamowski, 2013) for analysis for automatic road extraction from remote sensing (Benkouider et al., 2014). The demand forecasting using artificial neural networks (Slimani et al., 2015, 2017; Bousqaoui et al., 2018; Chawla et al., 2019) and optimized by artificial bee colony (Sultan and Jasim, 2016). The recurrent neural networks for sales prediction (Chen et al., 2018). Likewise, the comparison of forecasts based on neural networks vs. statistical methods. Hribar et al. (2019) make the comparison of models for forecasting the residential natural gas demand of an urban area.

The results achieved with the application of artificial neural networks in demand forecasting present these techniques as reliable in the development of forecasts and point to the feasibility of their establishment in industry. Where it is possible to emphasize that to implement the methodology ANN's, defined and trained, to determine the concentration of certain element of interest is a novel idea that is strengthened by the results. The entries to the network do not come only from data of the detected spectrum but they are based on the own antecedents of the experiment and are related with its different components (Marroquin et al., 2009).

In an effort to gain a better understanding of the use of artificial neural networks in production planning problems and to provide a basis for future research, a field observation study has been conducted on a metal working company in a principal city of zone North of Ecuador, managers and clients. Finding as the main problem of replenishment and non-compliance in the dispatch of marketed products.

\section{MATERIALS AND METHODS}

Currently, the use of artificial neural networks as a novel method and by virtue of the variant trend of planning in companies is displacing the more traditional statistical methods, especially because of the very promising results that are beginning to consolidate as a practical and dynamic model for decision making that is a very feasible option for the industry.

The proposed modeling is presented during the development of this research, in which the input variables that have already been provided by the company are presented, in addition to the conditioning factors that are indicated for weighting in each mathematical equation and the network in its autonomy will choose to use it or not followed by the hidden layers and the output layer (Claveria and Torra, 2014).

Due to the size of the data to be processed, the use of artificial intelligence is adequate and recommended, since, the feedback and dependence on processing generated by the neural network cannot be carried out by traditional statistics or at the same time, the level of confidence in the results would be affected.

The main quality of these systems is their dynamic adaptability, i.e., their ability to vary behavior in changing situations. To achieve this, they use techniques such as learning, generalization or self-organization. They are inspired by the model of a biological neuron using elemental processing units that mimic some of the characteristics of biological neurons.

The overall behavior of a network determines its ability to test hypothesis, detect statistical patterns and regularities or dynamically adjust an implicit model implemented in the same architecture. This behavior goes beyond the sum of the potentialities of the neurons that compose it. The result is the emergence of new properties that belong to the system.

Then, it is appropriate to use artificial intelligence to provide solutions to business problems because: artificial neural networks are a group of simulated neurons which are very interconnected, just like the neurons of the human brain and which are capable of learning in the same way as people do.

Neural networks architecture: The number of hidden neurons to be considered is achieved by a minimum default chosen as the initial optimal in MATLAB, choosing these 10 neurons which in turn translate into 10 iterations with 10 different combinations of weights without over-adjusting data or ignoring both linear and nonlinear feedback as shown in Fig. 1.

Supervised learning model: In fully supervised learning, centers, amplitudes and weights are determined in such a way as to minimize the error made by the network, so, it is not to be expected that the local behavior of the network will be preserved, as the overlap of neuron activation regions is not restricted.

To this end, it must be borne in mind that the dependence of the network output in relation to the 


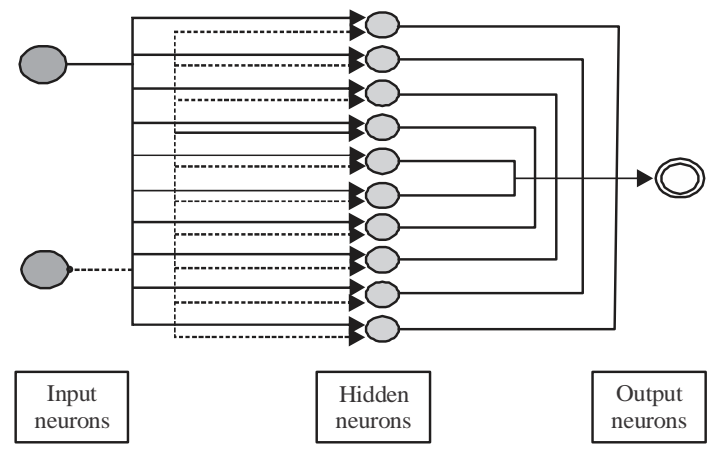

Fig. 1: ANN architecture

centers and amplitudes of the different neurons of the hidden layer is non-linear, so, non-linear optimization techniques must be used. The method of gradient descent is explained below. By means of this method we obtain the update of the parameters (centers, amplitudes and weights) that is carried out by means of the following equations:

$$
\begin{aligned}
& \mathrm{W}_{\mathrm{ik}}(\mathrm{n})=\mathrm{W}_{\mathrm{ik}}(\mathrm{n}-1)-\alpha_{1} \frac{2 \mathrm{e}(\mathrm{n})}{2 \mathrm{w}_{\mathrm{ik}}} \\
& U_{\mathrm{k}}(\mathrm{n})=U_{\mathrm{k}}(\mathrm{n}-1)-\alpha_{2} \frac{2 \mathrm{e}(\mathrm{n})}{2 \mathrm{u}_{\mathrm{k}}} \\
& \mathrm{C}_{\mathrm{ij}}(\mathrm{n})=\mathrm{C}_{\mathrm{ij}}(\mathrm{n}-1)-\alpha_{3} \frac{2 \mathrm{e}(\mathrm{n})}{2 \mathrm{c}_{\mathrm{ij}}} \\
& \mathrm{d}_{\mathrm{i}}(\mathrm{n})=\mathrm{d}_{\mathrm{i}}(\mathrm{n}-1)-\alpha_{4} \frac{2 \mathrm{e}(\mathrm{n})}{2 \mathrm{~d}_{\mathrm{i}}}
\end{aligned}
$$

Where:

$\mathrm{p}$ : The number of neurons in the input layer

$\mathrm{m}$ : The number of neurons in the hidden layer

$r$ : The number of output neurons

where, $\mathrm{j}=1,2, \mathrm{p}, \mathrm{i}=1,2, \ldots, \mathrm{m}$ and $\mathrm{k}=1,2, \ldots, \mathrm{r}$

Methodology for parameters selection: For this methodology all the introduced controlled parameters or the variables are taken as a test in this way we can have a control of the variations generated in the network (Fig. 2).

General algorithm of the system: This point analysis system generates information such as: training and test objective, training and test output, responses and error margins, allowing us to interpret and visually analyze all error results for each of the points of the variables



Fig. 2: Methodology for parameters selection with ANN Model

assigned to the study of this time series between the outputs and the objective pursued by the neural model, as shown (Fig. 3):

Forecast errors: To evaluate the forecast performance, error measurements will be used that compare the difference between the prediction and the actual value. MSE (Mean Square Error) is defined as the mean of $e_{t}^{2}$ this is the average of the errors between the estimator and what is estimated squared: 


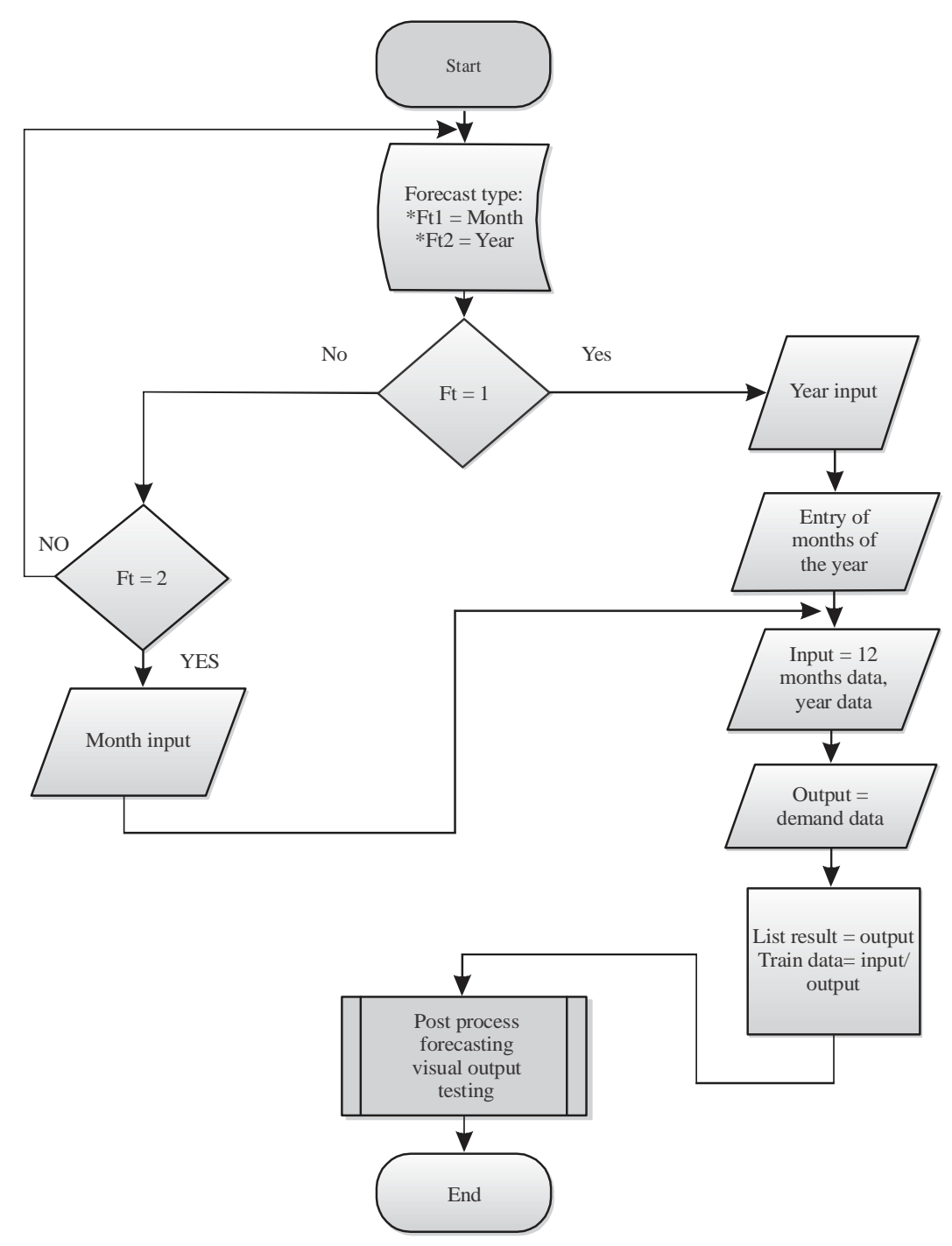

Fig. 3: System flowchart

$$
\text { MSE }=\frac{1}{\mathrm{n}} \sum_{\mathrm{t}=1}^{\mathrm{n}}\left(\mathrm{y}_{\mathrm{t}}-\hat{\mathrm{y}}_{\mathrm{t}}\right)^{2}
$$

Where:

$\mathrm{n}:$ The number of samples

$\hat{y}_{t}$ : The estimate of $y_{t}$

From the formula above, it follows that the measurement loss function is the quadratic or mean error squared. Correlation coefficient $\left(\mathrm{R}^{2}\right)$ where:

$$
\mathrm{R}^{2}=\frac{\frac{1}{\mathrm{n}} \sum_{\mathrm{t}=1}^{\mathrm{n}}\left(\mathrm{y}_{\mathrm{t}}-\hat{\mathrm{y}}_{\mathrm{t}}\right)^{2}}{\frac{1}{\mathrm{n}} \sum_{\mathrm{t}=1}^{\mathrm{n}}\left(\mathrm{y}_{\mathrm{t}}-\overline{\mathrm{y}}_{\mathrm{t}}\right)^{2}}
$$

Where:

$\hat{\mathrm{y}}$ : The forecasted demand

$y_{t}$ : The measured demand is the mean of the demand forecasting

n : The number of observations

Business diagnostics: It is logical to think that the installed capacity exceeds the demand. Idle capacity lowers prices until the most efficient find new stability, since, no one invests to improve efficiency in a product whose market is in extinction. The fundamental thing in these situations will be to continuously monitor sales and profitability, since, the latter will be the one that will tell us when to leave the market.

Principal products demand: Table 1-3 and Fig. 4 are given as. 


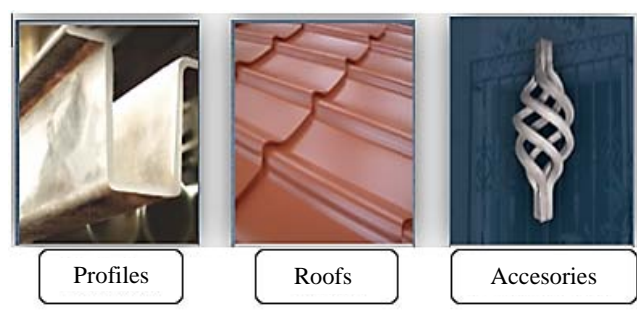

Fig. 4: Principal products

Table 1: Accessories demand (units)

\begin{tabular}{lrrrrr}
\hline Months & 2014 & 2015 & 2016 & 2017 & 2018 \\
\hline January & 2976 & 850 & 759 & 835 & 398 \\
February & 1245 & 860 & 742 & 468 & 456 \\
March & 2250 & 1245 & 945 & 295 & 832 \\
April & 6513 & 3049 & 3035 & 3032 & 923 \\
May & 6513 & 3049 & 3035 & 3032 & 923 \\
June & 978 & 1659 & 1163 & 687 & 469 \\
July & 1432 & 943 & 742 & 1002 & 451 \\
August & 6513 & 3049 & 3035 & 3032 & 923 \\
September & 963 & 1247 & 1005 & 541 & 398 \\
October & 562 & 945 & 785 & 235 & 398 \\
November & 562 & 860 & 1475 & 285 & 598 \\
December & 562 & 860 & 742 & 235 & 467 \\
\hline
\end{tabular}

Table 2: Profiles demand (units)

\begin{tabular}{llllll}
\hline Mounths & 2014 & 2015 & 2016 & 2017 & 2018 \\
\hline January & 2068 & 1731 & 1846 & 2237 & 587 \\
February & 2063 & 1777 & 1798 & 2152 & 496 \\
March & 2394 & 1657 & 1643 & 2098 & 601 \\
April & 2213 & 1621 & 1915 & 2331 & 551 \\
May & 2256 & 1920 & 1994 & 2312 & 524 \\
June & 2246 & 1624 & 1925 & 2381 & 489 \\
July & 2107 & 1744 & 2005 & 2624 & 548 \\
August & 2330 & 1787 & 2015 & 2489 & 579 \\
September & 2341 & 1648 & 1998 & 2156 & 497 \\
October & 2264 & 1660 & 1891 & 2265 & 602 \\
November & 2209 & 1634 & 1798 & 2605 & 583 \\
December & 2321 & 2078 & 1681 & 2392 & 405 \\
\hline
\end{tabular}

Table 3: Roofs demand (units)

\begin{tabular}{lccccc}
\hline Months & 2014 & 2015 & 2016 & 2017 & 2018 \\
\hline January & 1864 & 1244 & 1189 & 1036 & 549 \\
February & 1772 & 1388 & 1245 & 1089 & 481 \\
March & 1861 & 1225 & 1108 & 1167 & 505 \\
April & 1870 & 1442 & 1139 & 1217 & 518 \\
May & 1624 & 1194 & 1157 & 1094 & 579 \\
June & 1601 & 1541 & 1302 & 1176 & 399 \\
July & 1812 & 1533 & 1297 & 1099 & 476 \\
August & 1899 & 1340 & 1460 & 1284 & 603 \\
September & 2016 & 1496 & 1369 & 1005 & 548 \\
October & 2011 & 1564 & 1458 & 1187 & 586 \\
November & 2123 & 1182 & 1294 & 1108 & 475 \\
December & 2073 & 1425 & 1349 & 1126 & 403 \\
\hline
\end{tabular}

\section{RESULTS AND DISCUSSION}

The data provided by the company under study, shown in Table 1-3 are processed with the application of ANN and with the selection of the parameters for the development of the products forecast in a metalworking industry, the demand forecasting with the use of ANN and ARIMA for each one of the principal products is presented next.

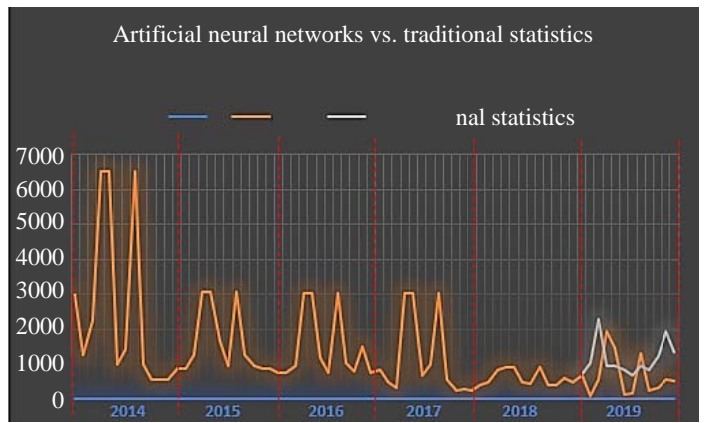

Fig. 5: Accessories forecasting comparison

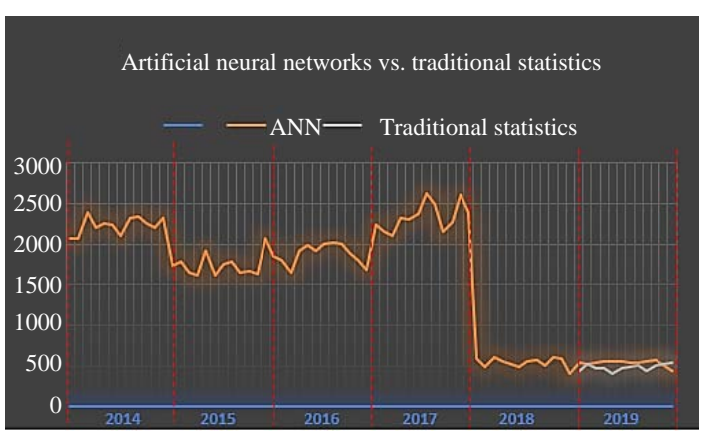

Fig. 6: Profiles forecasting comparison

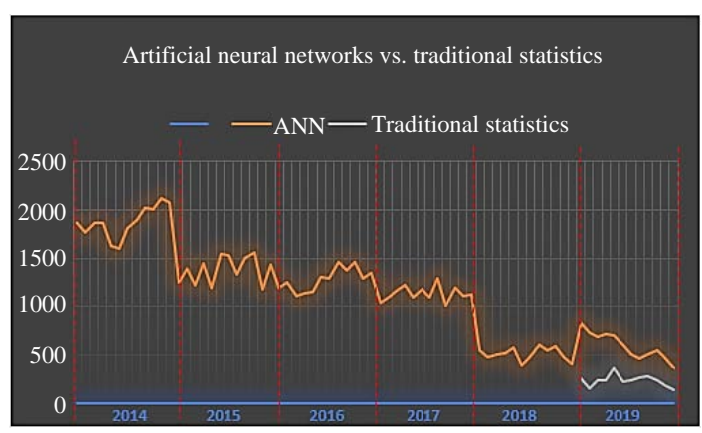

Fig. 7: Roofs forecasting comparison

\section{Accessories results:}

Profile results:

Roofs results: Figure 5-7 and Table 4-6 are given as.

Results comparison: The structure for the exact statistical method is ARIMA $(1,0,12)$ which means that you are describing some variable response $(\mathrm{Y})$ by combining a first-order automatic regression model and a 12-order moving average model because you work monthly. A good way to think about it is (AR, I, MA) (Tiwari and Adamowski, 2013).

The 0 between 1 and 12 represents the 'I' part of the model (the integrative part) and means a model in which the difference between the response variable data is being taken. This can be done with non-stationary data. The 
J. Eng. Applied Sci., 15 (1): 81-87, 2020

Table 4: Accessories demand forecasting (units)

\begin{tabular}{lcc}
\hline 2019 & Artificial neural networks & Traditional statistics \\
\hline January & 684 & 714 \\
February & 85 & 1012 \\
March & 537 & 2265 \\
April & 1908 & 946 \\
May & 1457 & 946 \\
June & 127 & 822 \\
July & 161 & 651 \\
August & 1305 & 944 \\
September & 232 & 837 \\
October & 305 & 1216 \\
November & 550 & 1944 \\
December & 517 & 1331 \\
\hline
\end{tabular}

Table 5: Profiles demand forecasting (units)

\begin{tabular}{lcc}
\hline 2019 & Artificial neural networks & Traditional statistics \\
\hline January & 546 & 440 \\
February & 520 & 520 \\
March & 531 & 470 \\
April & 559 & 479 \\
May & 564 & 410 \\
June & 553 & 464 \\
July & 542 & 481 \\
August & 540 & 498 \\
September & 551 & 436 \\
October & 566 & 502 \\
November & 498 & 523 \\
December & 446 & 546 \\
\hline & & \\
Table 6: Roofs demand forecasting (units) & \\
\hline 2019 & Artificial neural networks & Traditional statistics \\
January & 831 & 258 \\
February & 733 & 152 \\
March & 691 & 238 \\
April & 722 & 232 \\
May & 696 & 361 \\
June & 607 & 227 \\
July & 507 & 241 \\
August & 465 & 262 \\
September & 511 & 276 \\
October & 551 & 245 \\
November & 459 & 183 \\
December & 362 & 137 \\
\hline
\end{tabular}

statistical analysis test will be Ljung-Box as it fits these time series models and their randomness, on the other hand we cannot use the Durbin-Watson test as it is recommended for models with more than one delay and would not fit the current model as the analysis of Ljung-Box does so on the basis of one delay only. The Wald-Wolfowitz test is also not recommended, since, it does not comply with two characteristic parameters of this test which mention the comparison of two variables and in this model only one variable is analyzed. The second parameter is the verification of its independence which cannot be verified since the model only has one variable. Two main types of errors are compared where the MSE and the $\mathrm{R}^{2}$ error, the MSE delimits a range of $0-\infty$, that the smaller the MSE is the more adjusts to the real data is. On the other hand, the $\mathrm{R}^{2}$ delimits a range of $0-1$, being 1 the optimum (Table 7-9).
Table 7: Forecast comparison for accessories product

\begin{tabular}{lcc}
\hline Models & MSE & $\mathrm{R}^{2}$ \\
\hline Traditional statistics & 16.5 & 0.27 \\
ANN & 0.3 & 0.99 \\
\hline
\end{tabular}

Table 8: Forecast comparison for profile product

\begin{tabular}{lcc}
\hline Models & MSE & $\mathrm{R}^{2}$ \\
\hline Traditional statistics & 0.30 & 0.94 \\
ANN & 0.05 & 0.99 \\
\hline
\end{tabular}

Table 9: Forecast comparison for roofs product

\begin{tabular}{lcr}
\hline Models & MSE & $\mathrm{R}^{2}$ \\
\hline Traditional statistics & 0.30 & 0.89 \\
ANN & 0.06 & 0.99 \\
\hline
\end{tabular}

Once the evaluation of the errors has been carried out, artificial neural networks show a considerable advantage in the result of the proven errors having a noticeable advantage over traditional statistical methods.

\section{CONCLUSION}

With the revision of the techniques and algorithms used in the demand forecasting, the necessary foundation was obtained for the initial modeling of the neuronal structure. With the help of artificial neural networks, the behavior of the demand of the principal products of the metal-mechanic industry was diagnosed, obtaining the real behavior of the demand and the current planning. The artificial neuronal network model was constructed for the demand forecasting of the metal-mechanic industry.

With the comparison of the data predicted by means of artificial neural networks and traditional statistics, the validity was demonstrated by means of error indicators and the efficiency that the model has when forecasting by means of artificial neural networks.

\section{ACKNOWLEDGEMENTS}

The researcher acknowledge to the research project "Optimizacion de la Distribucion Fisica y en Planta en la Cadena de Suministroaplicando Tecnicas Heuristicas" supported by Agreement HCD Nro. UTN-FICA-20190149 by facultad de ingenieria en ciencias aplicadas from Universidad Tecnica del Norte. As well, researcher thank the valuable support given by the SDAS Research Group (www.sdas-group.com).

\section{REFERENCES}

Bellman, R.E. and L.A. Zadeh, 1970. Decision making in a fuzzy environment. Manage. Sci., 17: $141-164$. 
Benkouider, F., L. Hamami and A. Abdellaoui, 2014. Optimized neural networks using principal component analysis for automatic road extraction from remote sensing. J. Eng. Appl. Sci., 9: 427-433.

Bousqaoui, H., I. Slimani and S. Achchab, 2018. Information sharing as a coordination tool in supply chain using multi-agent system and neural networks. Proceedings of the 6th World Conference on Information Systems and Technologies (WorldCIST'18) Vol. 745, March 27-29, 2018, Springer, Naples, Italy, ISBN:978-3-319-77702-3, pp: 626.

Chawla, A., A. Singh, A. Lamba, N. Gangwani and U. Soni, 2019. Demand Forecasting Using Artificial Neural Networks-A Case Study of American Retail Corporation. In: Applications of Artificial Intelligence Techniques in Engineering: Advances in Intelligent Systems and Computing, Malik, H., S. Srivastava, Y.R. Sood and A. Ahmad (Eds.). Springer, Singapore, ISBN:978-981-13-1821-4, pp: 79-89.

Chen, C.F., M.C. Lai and C.C. Yeh, 2012. Forecasting tourism demand based on empirical mode decomposition and neural network. Knowl. Based Syst., 26: 281-287.

Chen, T., H. Yin, H. Chen, L. Wu and H. Wang et al., 2018. TADA: Trend alignment with dual-attention multi-task recurrent neural networks for sales prediction. Proceedings of the 2018 IEEE International Conference on Data Mining (ICDM), November 17-20, 2018, IEEE, Singapore, ISBN:978-1-5386-9160-1, pp: 49-58.

Claveria, O. and S. Torra, 2014. Forecasting tourism demand to Catalonia: Neural networks vs. time series models. Econ. Model., 36: 220-228.

Hribar, R., P. Potocnik, J. Silc and G. Papa, 2019. A comparison of models for forecasting the residential natural gas demand of an urban area. Energy, 167: 511-522.

Kim, S. and H. Kim, 2016. A new metric of absolute percentage error for intermittent demand forecasts. Intl. J. Forecasting, 32: 669-679.

Kochak, A. and S. Sharma, 2015. Demand forecasting using neural network for supply chain management. Intl. J. Mech. Eng. Rob. Res., 4: 96-104.
Lorente-Leyva, L.L., I.D. Herrera-Granda, P.D. Rosero-Montalvo, K.L. Ponce-Guevara and A.E. Castro-Ospina et al., 2018. Developments on solutions of the normalized-cut-clustering problem without eigenvectors. Proceedings of the 15th International Symposium on Neural Networks (ISNN 2018), June 25-28, 2018, Springer, Cham, Switzerland, ISBN:978-3-319-92536-3, pp: 318-328.

Marroquin, M.G.V., M.C.A. Cervantes, J.L.M. Flores and M. Cabrera-Rios, 2009. Time series: Empirical characterization and artificial neural network-based selection of forecasting techniques. Intl. Data Anal., 13: 969-982.

Rubio, J.D.J., I. Elias, D.R. Cruz, J. Pacheco and G.J. Gutierrez et al., 2017. A fuzzy algorithm for the prediction of future data. IEEE. Latin Am. Trans., 15: 1361-1367.

Sarmiento, AT. and O.C. Soto, 2014. New product forecasting demand by using neural networks and similar product analysis. Dyna, 81: 311-317.

Slimani, I., I.E. Farissi and S. Achchab, 2015. Artificial neural networks for demand forecasting: Application using Moroccan supermarket data. Proceedings of the 2015 15th International Conference on Intelligent Systems Design and Applications (ISDA), December 14-16, 2015, IEEE, Marrakech, Morocco, ISBN:978-1-4673-8709-5, pp: 266-271.

Slimani, I., I.E. Farissi and S. Achchab, 2017. Configuration and implementation of a daily artificial neural network-based forecasting system using real supermarket data. Intl. J. Logist. Syst. Manage., 28: 144-163.

Sultan, J.A. and R.M. Jasim, 2016. Demand forecasting using artificial neural networks optimized by artificial bee colony. Intl. J. Manage. Inf. Technol. Eng., 4: 77-88.

Tiwari, M.K. and J.F. Adamowski, 2013. Urban water demand forecasting and uncertainty assessment using ensemble wavelet-bootstrap-neural network models. Water Resour. Res., 49: 6486-6507.

Verma, A., A. Karan, A. Mathur and S. Chethan, 2017. Analysis of time-series method for demand forecasting. J. Eng. Appl. Sci., 12: 3102-3107. 